\title{
Características de los artículos publicados en las revistas "Matronas Profesión" y "Enfermería Nefrológica» durante los años 2001-2004
}

\author{
Gloria Seguranyes ${ }^{1,2}$ \\ Enriqueta Force ${ }^{3,4}$ \\ Carmen Fuentelsaz ${ }^{1,5}$ \\ Lola Andreu ${ }^{3,4}$ \\ Dolors Costa ${ }^{1,2}$
}

${ }^{1}$ Comité Editorial de "Matronas Profesión" ${ }^{2}$ Enfermera, Matrona. Unidad Docente de Matronas. TEU Escuela de Enfermería. Universidad de Barcelona ${ }^{3}$ Comité Editorial de "Enfermería Nefrológica"

${ }^{4}$ Enfermera. TEU Escuela de Enfermería.

Universidad de Barcelona

${ }^{5}$ Enfermera. Hospital «Vall d'Hebron». Barcelona

Este artículo se publica conjuntamente en las revistas Enfermería Nefrológica y Matronas Profesión, con autorización de las dos editoras de la revista.

\section{RESUMEN}

Objetivo. Conocer las características de los artículos publicados en las revistas Matronas Profesión (MP) y Enfermería Nefrológica (EN) durante los años 2001-2004.

Diseño. Estudio observacional, descriptivo y transversal.

Material y método. Las unidades de estudio fueron todos los artículos publicados durante estos 4 años en ambas revistas, excepto los editoriales. Se confeccionó una parrilla para efectuar el vaciado de las revistas. La recogida de los datos se realizó de manera independiente por dos personas para garantizar la fiabilidad de la información. Se recogió información sobre las características de cada uno de los artículos, de los autores y de las referencias bibliográficas. Sobre los artículos originales también se recogió el tipo de metodología utilizada, cuantitativa o cualitativa.

\begin{tabular}{|c|}
\hline Correspondencia: \\
Enriqueta Force \\
Escuela de Enfermería. Universidad de Barcelona \\
c/ Feixa Llarga s/n \\
08907 L'Hospitalet de Llobregat \\
Barcelona \\
Email: eforce@ub.edu
\end{tabular}

Resultados. Se han analizado 188 artículos, 80 $(42,6 \%)$ de MP y $108(57,4 \%)$ de EN. Los más frecuentes en EN han sido los artículos originales $(47,2 \%)$ y en MP los de formación continuada (36,3\%). El principal tema de estudio de EN ha sido la hemodiálisis $(41,7 \%)$ y de MP el parto (15\%). El número de autores por artículo es similar (4) en ambas revistas, así como las comunidades autónomas con más producción (Cataluña, Andalucía y Madrid), aunque con diferente distribución en ambas revistas.

Conclusiones. La revista EN presenta el mayor número de trabajos originales y la revista MP tiene una mayor calidad en el apartado de bibliografía. Cada una de las revistas presenta algunos aspectos que deberían mejorar para aumentar su calidad científica.

PALABRAS CLAVE: BIBLIOMETRÍA

ENFERMERÍA

MATRONAS

PUBLICACIONES CIENTÍFICAS 


\section{CHARACTERISTICS OF THE ARTICLES PUBLIS- HED IN «MATRONAS PROFESIÓN» AND «ENFER- MERÍA NEFROLÓGICA» FROM 2001 TO 2004}

\section{ABSTRACT}

Objective. To determine the characteristics of the articles published in "Matronas Profesión" (MP) and Enfermería Nefrológica" (EN) from 2001 to 2004.

Design. An observational, descriptive, cross-sectional study.

Material and methods. The study focused on all the articles, with the exception of editorials, published over those four years in the aforementioned journals. Access to the articles was attained, and the data was collected by two independent researchers in order to guarantee the reliability of the information. The characteristics of each article, the authors and the reference list were recorded. The original articles were characterized according to whether the methodology utilized was quantitative or qualitative.

Results. The authors analyzed 188 articles, 80 (42.6\%) from MP and 108 (57.4\%) from EN. Original articles predominated in $\mathrm{EN}$ (47.2\%), whereas articles focusing on continuing education were most common in MP (36.3\%). In $\mathrm{EN}$, the subject most frequently dealt with was hemodialysis $(41.7 \%)$ and in MP, childbirth (15\%). The mean number of authors per article was similar (4) in the two journals, as were the Spanish Autonomous Communities with the greatest production (Catalonia, Andalusia and Madrid), although the distributions differed from one journal to the other.

Conclusions. EN offers more original articles, while the quality of the reference lists is greater in MP. Both journals present certain aspects in which they should improve in order to increase their scientific quality.

KEY WORDS: BIBLIOMETRICS,

NURSING,

MIDWIFERY,

SCIENTIFIC PUBLICATION.

\section{INTRODUCCIÓN}

La profesión de enfermería, como otras que participan del conocimiento científico, progresa como resultado de la investigación en áreas que le son propias. Hasta finales de los años setenta, la investigación básica y aplicada en temas de enfermería fue muy escasa, y los profesionales se limitaban a colaborar en otras áreas de investigación biomédica, muchas veces realizando tareas mecánicas. Coincidiendo con la incorporación de los estudios de enfermería en la universidad y con la aplicación de políticas de calidad por parte del sistema sanitario, que obligan a hacer un seguimiento y evaluación de las actividades de enfermería, se inicia una actividad investigadora que da pie a la aparición de las primeras publicaciones en revistas propias de la disciplina. Desde entonces, se ha constatado un aumento progresivo de artículos de investigación en las revistas profesionales ${ }^{1-3}$.

La metodología bibliométrica es un instrumento esencial para evaluar el producto de la ciencia y, en el caso de disciplinas incipientes en materia de investigación, como es la enfermería, es una herramienta esencial para determinar su avance y estado de consolidación. La bibliometría es una línea de investigación en la que se ha dedicado mucha atención en los últimos años y son numerosas las publicaciones que sobre este tema han realizado varios autores ${ }^{46}$. La mayoría de los estudios se centran en el análisis de las tres revistas españolas de la disciplina enfermera con mayor impacto (ROL, Enfermería Científica y Enfermería Clínica), a las que en los últimos años se han sumado Index de Enfermería y Metas de Enfermería ${ }^{8}$.

Desde el nacimiento de las primeras revistas de enfermería en el Estado español hasta nuestros días, el número de publicaciones periódicas se ha incrementado considerablemente $e^{9}$; además de las revistas que dependen de empresas editoriales, hay otras promovidas por centros asistenciales y universidades, colegios de enfermería y asociaciones científicas. La primera revista española de matronas científico profesional se publicó, con carácter regular, entre los años 1980 y $2001^{10}$.

Las dos revistas objeto de este estudio son Enfermería $\mathrm{Ne}$ frológica y Matronas Profesión.

Matronas Profesión se inició en septiembre de 2001. Tiene una periodicidad trimestral y su contenido es de tipo científico y profesional, tiene una tirada de 5.000 ejemplares y se distribuye de forma gratuita a las matronas asociadas del Estado español y a aquellas que lo solicitan. Se suministra, asimismo, a las bibliotecas de escuelas de enfermería y a las unidades docentes de matronas. La financiación de la revista se realiza por cuatro patrocinadores, que se hacen cargo del total de los costes.

En la revista hay dos apartados diferenciados: el contenido científico y el de divulgación profesional. En el primero, los diferentes tipos de artículos que se publican son: editoriales, artículos originales, de revisión, formación continuada, protocolos de actuación, planes de cuidados, informes, y un artículo especial que incluyen los patrocinadores. En el apartado de divulgación profesional se incluye una entrevista, la vida de las asociaciones, con información sobre las actividades que realizan, y la agenda, con la información referente a congresos, cursos, premios, etc. 
La revisión de cada uno de los artículos (originales o no) se efectúa por dos personas expertas del comité editorial, del comité científico o por evaluadores externos. El comité científico está formado por representantes de las 16 asociaciones de matronas del Estado español.

La Revista de la Sociedad Española de Enfermería Nefrológica, en su actual formato, se publica desde 1998, pero representa la continuidad de otra denominada BISEAN, que fue el primer órgano de difusión de la Sociedad Española de Enfermería Nefrológica, fundada en 1975 con el objetivo de promover el progreso científico y el intercambio de experiencias, con vistas a lograr el mejor cuidado de los enfermos renales.

La revista tiene una periodicidad trimestral y consta de dos apartados, uno que corresponde al contenido científico y otro relacionado con las actividades de la Sociedad. El apartado científico incluye: editorial, originales, formación continuada, casos clínicos, planes de cuidados y bibliografía comentada. En el otro apartado se incluye: entrevistas, información sobre cursos, congresos, premios, becas y otros aspectos divulgativos. La revisión del contenido se realiza por pares. Tiene una tirada de 3.000 ejemplares, se distribuye gratuitamente entre los asociados, colaboradores y bibliotecas de ciencias de salud de toda España y algunos países iberoamericanos. La financiación es en un 30\% mediante publicidad y el resto a cargo de la Sociedad Española de Enfermería Nefrológica.

Ambas revistas están indexadas en las bases de datos Cuiden, Cuidatge, BDIE, Latindex, IBECS y Enfispo. Además, Enfermería Nefrológica lo está en CINAHL y también hay una versión electrónica disponible a texto completo en la página web de la Sociedad y en la base de datos Scielo.

A raíz de la I Conferencia Iberoamericana de Editores de Revistas de Enfermería, que se celebró en Granada en el año 2003, se valoró la importancia de hacer estudios bibliométricos para conocer la evolución de las revistas, y difundir los resultados en congresos o en publicaciones científicas. Por ello, se planteó realizar el presente estudio sobre las dos revistas, dadas sus similitudes en cuanto a periodicidad, extensión, distribución y áreas de estudio especializadas.

El objetivo general es conocer las características de los artículos publicados en ambas revistas durante los años 2001-2004. Como objetivos específicos se han planteado: identificar el tipo de artículos publicados, según su estructura y contenido, y conocer las características de los artículos originales publicados en ambas revistas.

\section{MATERIAL Y MÉTODO}

Diseño. Estudio observacional, descriptivo y transversal.

Ámbito. La publicación de las revistas Matronas Profesión (MP) y Revista de la Sociedad Española de Enfermería $\mathrm{Ne}$ frológica (EN), desde enero de 2001 a diciembre de 2004, ambos inclusive.

Material. Todos los artículos publicados en ambas revistas durante los 4 años de estudio. Se han incluido todos los artículos, excepto los editoriales y los artículos encargados por los patrocinadores de la revista MP.

\section{Variables}

Respecto a las revistas, se han recogido las siguientes variables: identificación de la revista, año, volumen y número de la publicación.

Respecto a las características de cada uno de los artículos, las variables estudiadas han sido: tipo de artículo publicado (artículo original, original breve, formación continuada, caso clínico, protocolo de actuación, plan de cuidados, informe, otros), número de páginas y tema de estudio.

Sobre los autores se recogieron los siguientes datos: número, género, ámbito de trabajo, comunidad autónoma a la que pertenece y titulación del primer autor, y si el trabajo era pluridisciplinario (sí, no).

Por lo que respecta a la bibliografía, se contemplaron las siguientes variables: número de referencias, procedencia (estatal o internacional), y actualidad de las citas bibliográficas, así como las características de los documentos citados.

Además, sobre los artículos originales se analizó el tipo de metodología utilizada (cualitativa, cuantitativa o ambas).

\section{Recogida de datos}

Se confeccionó una parrilla para efectuar el vaciado de las revistas. La recogida de los datos se realizó de manera independiente por dos personas para garantizar la fiabilidad de la información.

\section{Análisis de los datos}

Se ha efectuado un análisis descriptivo para todas las variables, las cuantitativas se han descrito con media y desviación estándar (DE), si seguían una distribución normal, o con mediana, mínimo y máximo en caso contrario. Las cualitativas, con frecuencias absolutas y porcentajes de cada una de las categorías. 
Para la comparación de proporciones se utilizó la prueba de la ji al cuadrado $\left(\mathrm{x}^{2}\right)$ o la prueba exacta de Fisher, y para la comparación de medias la t de Student o la U de Mann-Whitney. Se estableció un nivel de confianza del 95\% para todos los casos. Se utilizó el paquete de programas estadísticos SPSS 12.0 .

\section{RESULTADOS}

Se han estudiado 188 artículos, 80 (42,6\%) de MP y 108 $(57,4 \%)$ de EN. El tipo de artículos de las revistas MP y EN, publicados entre los años 2001 y 2004 , se presenta en las tablas 1 y 2 , respectivamente; los más frecuentes fueron los de formación continuada en MP $(29 ; 36,3 \%)$ y los artículos originales $(51 ; 47,2 \%)$ en EN.

\begin{tabular}{|l|c|l|}
\hline Tipo de artículo & Frecuencia absoluta & $\mathbf{\%}$ \\
\hline Formación continuada & 29 & 36,3 \\
\hline Original & 19 & 23,8 \\
\hline Protocolo de actuación & 15 & 18,8 \\
\hline Revisión & 11 & 13,8 \\
\hline Otros & 6 & 7,5 \\
\hline
\end{tabular}

Tabla 1. Tipo de artículos de la revista Matronas Profesión.

\begin{tabular}{|l|c|l|}
\hline Tipo de artículo & Frecuencia absoluta & $\mathbf{\%}$ \\
\hline Original & 51 & 47,2 \\
\hline Original breve & 17 & 15,7 \\
\hline Caso clínico & 15 & 13,9 \\
\hline Protocolo de actuación & 7 & 6,5 \\
\hline Plan de cuidados & 6 & 5,6 \\
\hline Formación continuada & 6 & 5,6 \\
\hline Otros & 6 & 5,6 \\
\hline
\end{tabular}

Tabla 2. Tipo de artículos de la revista Enfermería Nefrológica.

La media del número de páginas de los artículos de la revista MP es de 6,3 $(\mathrm{DE}=2,1)$ y de 4,4 $(\mathrm{DE}=1,9)$ para la revista $\mathrm{EN}$. Los principales temas de estudio de los trabajos publicados se reflejan en las tablas 3 (MP) y 4 (EN).

La mediana del número de autores por artículo en MP fue de $2(\min =1, \max =8)$, y en EN de $4(\min =1, \max =13)$. En relación con el género del primer autor, el 71,3\% (57) eran mujeres en MP, y el 67,6\% (73) en EN.

El ámbito de trabajo de los primeros autores de MP era el hospital $(25 ; 31,3 \%)$, en docencia trabajaban 24 (30\%) y en atención primaria 18 (22,5\%). En la revista EN, la mayoría (103; $95,4 \%)$ trabajaba en el hospital, $3(2,8 \%)$ en docencia y 1 $(0,9 \%)$ en atención primaria.

\begin{tabular}{|l|c|l|}
\hline Tema de estudio & Frecuencia absoluta & $\mathbf{\%}$ \\
\hline Parto & 12 & 15 \\
\hline Posparto & 9 & 11,3 \\
\hline Educación sanitaria & 8 & 10,1 \\
\hline Investigación & 7 & 8,8 \\
\hline Ejercicio profesional & 6 & 7,5 \\
\hline
\end{tabular}

Tabla 3. Principales temas de estudios publicados en la revista Matronas Profesión.

\begin{tabular}{|l|c|l|}
\hline Tema de estudio & Frecuencia absoluta & $\mathbf{\%}$ \\
\hline Hemodiálisis & 45 & 41,7 \\
\hline Diálisis peritoneal & 13 & 12 \\
\hline Accesos vasculares & 10 & 9,3 \\
\hline Nutrición & 6 & 5,6 \\
\hline Trasplante renal & 5 & 4,6 \\
\hline
\end{tabular}

Tabla 4. Principales temas de estudios publicados en la revista Enfermería Nefrológica.

Las comunidades autónomas de procedencia más frecuente de los primeros autores en MP fueron Cataluña (25; 31,3\%), seguida de Andalucía (20; 25\%) y Madrid (11; 13,8\%). En el caso de EN, fueron las de Andalucía (23; 21,3\%), Cataluña (21; $19,4 \%)$ y Madrid (19; 17,6\%). La titulación de Matrona es la más frecuente $(61 ; 76,3 \%)$ entre los autores de MP, y la de Enfermería $(101 ; 93,5 \%)$ en la revista EN.

Se han realizado de forma pluridisciplinaria el 21,3\% (17) de los trabajos de MP y el 15,7\% (17) de los de EN.

El número y las características de las referencias bibliográficas de ambas revistas se describen en la tabla 5.

\begin{tabular}{|c|c|c|c|c|c|c|}
\hline \multirow[b]{2}{*}{ Referencias } & \multicolumn{3}{|c|}{ Matronas Profesión } & \multicolumn{3}{|c|}{ Enfermería Nefrológica } \\
\hline & Mediana & Mínimo & Máximo & Mediana & Mínimo & Máximo \\
\hline Número & 15,5 & 0 & 69 & 7 & 0 & 57 \\
\hline Nacionales & 6 & 0 & 42 & 3 & 0 & 16 \\
\hline Internacionales & 8 & 0 & 69 & 2 & 0 & 45 \\
\hline Revistas & 9 & 0 & 55 & 3 & 0 & 52 \\
\hline$\leq 2$ años & 4 & 0 & 22 & 0,5 & 0 & 9 \\
\hline$>2,<5$ años & 3,5 & 0 & 20 & 2,5 & 0 & 24 \\
\hline$>5$ años & 7 & 0 & 42 & 3 & 0 & 30 \\
\hline
\end{tabular}

Tabla 5. Características de las referencias bibliográficas de los artículos publicados en las revistas Matronas Profesión y Enfermería Nefrológica.

En relación con los artículos originales (AO), se han publicado más en la revista EN $(51 ; 47,2 \%)$ que en MP $(19 ; 23,8 \%)$, siendo esta diferencia estadísticamente significativa $\left(\mathrm{x}^{2}=\right.$ $10,83 ; \mathrm{gl}=1 ; \mathrm{p}=0,001)$. 
La metodología utilizada en los $\mathrm{AO}$ de ambas revistas ha sido, mayoritariamente, de tipo cuantitativo: 18 (94,7\%) en MP y 61 (89,7\%) en EN. En un artículo original de MP se utilizó metodología cualitativa, y en 4 de EN. En esta última se utilizó metodología mixta en $3 \mathrm{AO}$.

El tema estudiado con mayor frecuencia en los AO de ambas revistas, coincide con el tema de estudio del resto de los otros tipos de artículos publicados.

Las comunidades autónomas de procedencia más frecuente del primer autor de los 19 AO publicados en MP fueron Andalucía 6, Madrid 5, Cataluña 3 y Castilla La Mancha 2. De los 51 AO publicados en EN, 10 eran de Andalucía, 10 de Cataluña, 7 de Madrid y 5 de cada una de las comunidades autónomas de Aragón y Valencia.

En la tabla 6 se presentan las características de los AO de ambas revistas. Se han encontrado diferencias estadísticamente significativas entre las dos revistas, y las medianas eran más elevadas en MP que en EN, en relación con el número de referencias bibliográficas totales, internacionales, revistas y antigüedad $\leq 2$ y $>5$ años.

\begin{tabular}{|c|c|c|c|c|c|c|c|c|}
\hline \multirow[b]{2}{*}{ Referencias } & \multicolumn{3}{|c|}{ Matronas Profesión } & \multicolumn{3}{|c|}{ Enfermería Nefrológica } & \multicolumn{2}{|c|}{ Significación estadística } \\
\hline & Mediana & Mínimo & Máximo & Mediana & Mínimo & Máximo & $\begin{array}{c}\mathrm{U} \\
\mathrm{de} \\
\text { Mann- } \\
\text { Whitney }\end{array}$ & $\mathrm{p}$ \\
\hline Número & 17 & 11 & 34 & 9 & 3 & 57 & 157 & $<0,001$ \\
\hline Nacionales & 6 & 0 & 34 & 5 & 0 & 16 & 382,5 & 0,176 \\
\hline Internacionales & s 8 & 0 & 26 & 3 & 0 & 45 & 272 & 0,005 \\
\hline Revistas & 13 & 2 & 23 & 6 & 0 & 52 & 205,5 & $<0,001$ \\
\hline$\leq 2$ años & 4 & 0 & 20 & 1 & 0 & 7 & 146,5 & $<0,001$ \\
\hline$>2, \leq 5$ años & 4 & 1 & 13 & 3 & 0 & 24 & 404 & 0,281 \\
\hline$>5$ años & 9 & 1 & 17 & 4 & 1 & 30 & 254,5 & 0,002 \\
\hline $\begin{array}{l}\text { Número de } \\
\text { autores }\end{array}$ & 4 & 1 & 7 & 4 & 1 & 11 & 464,5 & 0,790 \\
\hline
\end{tabular}

Tabla 6. Características de los artículos originales publicados en Matronas Profesión y Enfermería Nefrológica.

\section{DISCUSIÓN}

En la revista MP el tema de estudio más frecuente ha sido el parto, el tema más estudiado también en las décadas de los ochenta y noventa en nuestro país, según refleja la producción científica relacionada con las matronas ${ }^{11}$.

El ámbito de trabajo más frecuente de los autores se ha distribuido entre el hospital y la docencia, y en segundo lugar el de atención primaria, lo que coincide con otros estudios realiza$\operatorname{dos}^{11,12}$. Esta amplia participación de la matronas docentes puede deberse a la incorporación progresiva de matronas a las unidades docentes en estos últimos años. Asimismo, en el ma- pa bibliométrico de España de biomedicina y ciencias de la salud, la producción científica mayoritaria proviene del ámbito universitario ${ }^{13}$.

A pesar de la inclusión de la materia de investigación en el nuevo modelo formativo de matronas en 1994, el porcentaje de artículos originales publicados en la revista MP no alcanza al indicado por diversos autores ${ }^{2,14}$. Estos datos concuerdan con un estudio publicado en España, que muestra que sólo un $13 \%$ de las matronas que se formaron entre el año 1994 y 2003 había publicado trabajos de investigación ${ }^{15}$. En el estudio realizado por el American College of Nurse-Midwives sobre las tareas profesionales que hacían las matronas se observa que, mientras las recién graduadas manifestaban que la investigación era una tarea importante, cuando se les preguntaba si la realizaban la respuesta afirmativa fue muy baja ${ }^{16}$. Por otra parte, una de las causas de los pocos trabajos de investigación que se publican, a pesar de tratarse de una revista específica, puede ser la escasez de matronas en España desde la década de los noventa ${ }^{17}$.

En la revista EN destaca que la mayor parte de los artículos son originales, fruto de la larga tradición investigadora de la enfermería nefrológica que, desde sus inicios, ha tenido que enfrentarse a la resolución de nuevos problemas, relacionados con la complejidad del tratamiento de la insuficiencia renal. La fundación de la revista coincidió con una etapa de experimentación clínica en que las técnicas de sustitución de la función renal y el trasplante estaban en sus inicios en nuestro país (la primera hemodiálisis se llevó a cabo en Barcelona en 1957 y el primer trasplante de riñón en 1965$)^{18}$, lo que obligó a la enfermería, ante los nuevos retos que se le presentaban, a experimentar e investigar. El resultado de la investigación en enfermería nefrológica se presenta en los congresos anuales de la Sociedad y en los últimos años se publican en EN todos los trabajos que han obtenido premios en dichos congresos, lo que nos permite disponer de una importante fuente de documentación.

En cuanto a los temas tratados en los artículos originales prevalecen los relacionados con la hemodiálisis o técnicas similares y la diálisis peritoneal, lo que refleja la actividad asistencial de la enfermería nefrológica, que se centra, básicamente, en prestar cuidados a las personas con insuficiencia renal crónica que están en programas de sustitución de la función renal $^{19,20}$. Estos programas de atención se basan en la realización de técnicas muy sofisticadas, que deben realizarse con mucha frecuencia y durante largos periodos de tiempo, por lo que la enfermería no sólo debe resolver los múltiples problemas técnicos que se plantean sino que, además, ha de prestar una atención integral a las personas sometidas a tratamiento, la cual incluye educación, soporte emocional al paciente y la familia y todo lo que implica una visión holística de la persona. Hemos de destacar que aunque el trasplante renal es una forma habitual de tratamiento de la insuficiencia 
renal, y que actualmente nuestro país figura entre los primeros del mundo en número y buenos resultados ${ }^{21}$, esto no se refleja en el número de artículos que sobre este tema se han publicado en la revista.

El ámbito de trabajo de los autores es eminentemente hospitalario (en este apartado, se han incluido los centros de diálisis dependientes de hospitales), en relación con la actividad asistencial que desarrolla; no obstante, se debe tener en cuenta que en las consultas ambulatorias de los hospitales, se presta atención a pacientes renales, y en ellas la enfermería nefrológica tiene un papel muy relevante $e^{22}$, por lo que trabajos realizados en las consultas de enfermería, aunque hospitalarios, tienen un alto componente de asistencia primaria.

Respecto al número de autores de los artículos originales, ambas revistas tienen la misma mediana, similar a la encontrada en otros trabajos ${ }^{12,14}$. La revista EN tiene algunos artículos con más autores, lo que puede deberse a que los servicios de nefrología están formados por profesionales sanitarios que trabajan en equipos muy definidos, y todos sus miembros participan de los trabajos de investigación. Esto también explicaría que aunque mayoritariamente los autores son enfermeros, en algunos se incluyen otros profesionales, como médicos, psicólogos o dietistas.

Prevalece el género femenino en el primer autor, lo que refleja la realidad social del colectivo enfermero; sin embargo, en ambas revistas el porcentaje de primeros autores hombres supera el habitual de nuestra profesión. Según el Instituto Nacional de Estadística (INE), el porcentaje de enfermeros masculinos colegiados en el año 2004 era del 22,53\% ${ }^{23}$. Esto concuerda con el hecho de que los hombres eligen con frecuencia la especialidad de la enfermería nefrológica, ya que ofrece aspectos atractivos para ellos, como su complejidad técnica. En la Sociedad Española de Enfermería Nefrológica dicha relación es del $31 \%{ }^{24}$.

Como ya se ha descrito en otros estudios bibliométricos ${ }^{12,13}$, las comunidades autónomas que más producción científica han aportado a ambas revistas son Andalucía, Cataluña y Madrid, que son las que tienen un mayor número de matronas en España; por otra parte, son las que cuentan con mayor infraestructura hospitalaria absoluta y mayor número de centros de diálisis $y$, por tanto, es donde se atiende a un mayor número de enfermos renales.

Prevalece la investigación cuantitativa, que se lleva a cabo en los estudios experimentales y descriptivos. Con este tipo de estudios la enfermería se introdujo en la investigación, siguiendo el modelo biomédico que imperaba en la atención sanitaria. Más recientemente, la mayor autonomía de la enfermería -y, por tanto, de la enfermería nefrológica- la ha llevado a inte- resarse por temas más específicos de su rol, para los que se ha utilizado tanto una metodología cuantitativa como cualitativa, según el problema de estudio planteado.

Donde sí se aprecian diferencias entre ambas revistas es en las referencias bibliográficas, tanto en los artículos originales como en el total de estudios -la revista MP es la que presenta más referencias, tanto nacionales como internacionales- y en cuanto a su actualidad. Por tanto, éste es un aspecto que debería mejorar la revista EN, donde además, se citan con mayor frecuencia libros que revistas, y esto puede implicar una menor actualización. Las razones que pueden influir en una mayor citación en el contexto de las matronas pueden ser el énfasis realizado en la formación continuada sobre revisiones bibliográficas y también la incorporación de los nuevos profesionales con formación específica de investigación. Por otra parte, la necesidad prioritaria de la revista MP es atraer más trabajos originales en el ámbito de la salud sexual y reproductiva para su publicación.

\section{BIBLIOGRAFÍA}

1. Torra JE. Producción científica de la enfermería española. Un análisis a través de los artículos publicados en tres revistas durante el trienio 1991-1993. Rev Rol Enferm. 1995; (198): 61-71.

2. Richart M. Estado de la producción científica de la enfermería española (I). Index Enferm. 1999; 8 (27):19-24.

3. Delicado MV, Pardo C, Reolid M, García MJ, Mallebrera E. Investigación en enfermería: adecuación de la estructura de los originales publicados en la década 1985-1994. Enferm Clin. 2001; 11: 201-13.

4. Icart MT, Gracia S, Pulpón AM, Fuentelsaz C, López S, Pedreny R. Introducción y análisis estadístico en los originales publicados en dos revistas de enfermería y en dos de medicina (1995-1996). Enferm Clin. 1999; 9: 55-60.

5. Pardo C, Mallebrera E, García MJ, Reolid M, Delicado MV. Características de la producción científica en enfermería en la década 1985-1994. Enferm Clin. 2001; 11: 51-64.

6. Gálvez A. Un modelo explicativo de la pauta de citación a revistas de enfermería españolas. Enferm Clin. 2002; 12 : 210-6.

7. Gálvez A. Publicaciones de enfermería en España. Rev Rol Enferm. 2001; 24: 355-9.

8. Gálvez A, Hueso C, Amezcua M. Indicadores CUIDEN de repercusión de las revistas de enfermería del área lingüística del español y del portugués. Index Enferm Digital. 2004; 46 [Acceso el 25 de abril de 2006]. Disponible en: http://www.index-f.com/index-enfermeria/46revista/46_articulo_76-80.php>

9. Serrano P, Giménez AM, Arroyo MP. Análisis de la producción científica publicada en la revista Metas de Enfermería. Index Enferm. 2005; 14(48-49): 78-82. 
10. Fernández M, Sánchez MI, Blas M, Blaya N, Casado E, Duran $\mathrm{M}$, et al. Estudio bibliométrico de las publicaciones de matrona durante el periodo 1980-1995. Matronas Hoy. 1999; (12): 15-24.

11. Sánchez MI, Fernández M. Producción científica relacionada con la especialidad de matrona. Metas Enferm. 2000; 3(23): 35-41.

12. Richart M. Estado de la producción científica de la enfermería española (y II). Index Enferm. 2000; 9(28-29): 158.

13. Cami J, Suñen-Piñol E, Méndez-Vázquez R. Med Clin (Barc). 2005; 124(3): 93-101.

14. Pérez C, Estrada JM, Villar F, Rebollo MJ. Estudio bibliométrico de los artículos originales de la Revista Española de Salud Pública (1991-2000) (I): indicadores generales. Rev Esp Salud Pública. 2002; 76(6): 659-72.

15. Sánchez MI. Situación profesional de las nuevas matronas. Matronas Prof. 2003; 4(13): 23-8.

16. Johnson PG, Oshio S, Fisher MC, Fullerton JT. The 1999-2000 ACC task analysis of nurse-midwifery/midwifery practice: a consideration of the concept of professional issues. J Midwifery Womens Health. 2001; 46(5): 313-20.
17. Seguranyes G. Escasez de matronas. Matronas Prof. 2002; (8): 3.

18. Andreu L, Force E. La enfermería y el trasplante de órganos. México: Editorial Médica Panamericana; 2004.

19. Zampieron A, Harrington M, Elseviers MM, Lindeley E, De Vos JY, Visser R. The Research Board of the EDTNA/ERCA. EDTNA/ERCA Journal. 2004; 30(2): 59-61.

20. De Vos JY, Elseviers MM, Harrington M, Zampieron A, Vlaminck H, Ormandy P, et al. Práctica europea en HD: resultados de la EPD. EDTNA/ERCA Journal (ed. esp.). 2006; 32(1): 21-4.

21. Deulofeu R. Activitat de trasplantaments. Butlletí de Trasplantament. 2005; 32: 2-5.

22. Martín Espejo JL, Guerrero Riscos MA. Qué demanda el paciente de prediálisis de la consulta de enfermería. Nuestra experiencia. Rev Soc Esp Enf Nefrol. 2002; 2ํㅜ trimestre $\mathrm{n}^{\circ}$ 18: 17-21.

23. INE. Profesionales sanitarios colegiados 2004 [Acceso 2 mayo 2006]. Disponible en: http://www.ine.es/inebase $/$ cgi $/$ um? $\mathrm{L}=\& \mathrm{~N}=\& \mathrm{O}=$ pcaxis $\& \mathrm{M}=\% 2 \mathrm{Ft} 15 \% 2 \mathrm{Fa} 041 \%$ 2Fa2004

24. Andreu L, Guillén A. Memoria de la Sociedad Española de Enfermería Nefrológica. Madrid: SEDEN; 2000. 